\title{
Impact of Bio-statistics in Medical Sciences
}

\author{
*Mobin Ahmad \\ Department of Mathematics, Faculty of Science, Jazan University, Saudi Arabia \\ *Corresponding Author *PV Sampath Kumar
}

\begin{abstract}
Biostatistics is undesirably seen as a mathematical science. Time now is to reorient the subject with the goal that it makes the most of its legitimate place into medical disciplines as opposed to being dealt with as an outsider subject. The accompanying is a concise blueprint of the arrangement of showing this subject can be extremely persuading to medical undergraduates as the one that satisfies their medical need. In the same way as other fields, public health has grasped the procedure of confirmation based practice to illuminate rehearse choices and to guide arrangement advancement. Prove construct practice is regularly needy upon speculations made with respect to the bases of the current group of learning - osmoses of the exploration writing on a specific subject. The potential utility of logical confirmation for managing strategy and practice choices is grounded in the legitimacy of the exploration examinations whereupon such choices are made. Nonetheless, the legitimacy of inductions produced using the surviving public health research literature requires more than finding out the legitimacy of the bio-statistical methods alone; for each review, the legitimacy of the whole research prepare must be fundamentally examined to the best degree conceivable so that proper conclusions can be drawn, and that suggestions for advancement of sound public health policy and practice can be offered. Bio-statistics has been requested progressively in biomedical research since the 1960 s for support in experimental design, mathematical and biostatistician modeling and additionally computational biostatistics. This brought about the foundation of bio-statistical units in colleges, investigate organizations and bigger pharmaceutical companies; an advancement not without unsettling influences and routinely reexamining the part of biostatistics and its avocation at that spots. This paper presents an approach where counseling and skill is joined with participation, on the premise of sound biostatistical science and original bio-statistical research.
\end{abstract}

Keywords: Bio-statistics, Medical Sciences, public health, process, development, policy, research, experimental design, mathematical, statistical modeling, pharmaceutical companies.

\section{Introduction:}

The field of biostatistics has been developing since around the 1960s, when biostatisticians were requested for the utilization of mathematical and statistical methods and procedures in biological and biomedical sciences. In Germany, this improvement was started in 1960 by proposals of a Science Committee expressing the vitality of medical biostatistics and information documentation for medical research. This activity was authorized later (1973) by executing an educational programs on Biomathematics for restorative understudies. Another push forward came for biostatistics in 1976 when another medication law requested greater quality, viability and wellbeing for clinical studies, rendering accepted the use of bio-statistical standards crucial. As a result bio-statistical science developed in numerous nations as a teach, alluding to the 'application of biostatistics, probability, computing and mathematics to the life sciences, with the objective of progressing our knowledge'.

Rethinking the part of a bio-statistical unit at a research center is essential when its reality is addressed now and again by audit boards of trustees, by managerial bodies, by financial supports, or by companion associates in or outside the foundation. Scrutinizing the need or freedom of biostatistics turns into an issue when financial resources turn out to be short or when new biomedical branches begin to contend in bio-statistical themes, or when it winds up noticeably stylish to rebuild a foundation. At that point it ends up plainly basic to clarify the idea of biostatistics to researchers and partners. One additionally needs to contend for the justification of the teach in a research center with regards to the opposition on assets. To be clear about the documentation: We will utilize the two terms biostatistics and biometry interchangeable independent of a few talks on separating between biometry and biostatistics [1]. Really, our department got its name right around 30 years ago when it was changed from Experimental Design to Biostatistics.

\section{Review of literature:}

The need for prove based basic leadership in public health has developed in unmistakable quality amid late years. In the mid-1990s, when the principal endeavors were embraced to blend logical data about the viability of health promotion and illness counteractive action programs, few public health professionals and policymakers knew about the thought of confirmation based practice. Presently, over 10 years after the fact, 
topics of evidence-based public health (EBPH) have turned into the concentration of regional, national, and international public health meeting motivation, and the expression "proof of viability" is a focal topic of public health practice. EBPH is grounded in the confirm based practice development in the field of medicine. In any case, there are prominent contrasts between the two orders of drug and public health that require unmistakable methodologies of the use of confirmation based practice. In particular, the objective of confirm based medicine is "... the best possible management of health and disease in singular patients... " The goal in EBPH is "... the most ideal administration of health and disease and their determinants at the group level." As such, policy development and intercessions to enhance the health of our public require a comprehension of the complexities of organizational structures, communications, and a heap of different elements that effect basic leadership at the local, state, regional, and national levels.

In today's public health commercial center, we have been made a request to fabricate a practice that is the combination of logical aptitudes, improved correspondence, political discernment, and sound judgment, yet, how best to direct assessment look into or potentially to decipher inquire about discoveries in the "prescribed procedures" world in which we now live has been surrendered to a great extent over to scientists or potentially governmental agencies. As a result of the staggering volume of research writing, it has been hard to deal with and separate what is compelling for's one of a kind public health practice needs. In spite of the fact that the Guide to Clinical Preventive Services [2] and the Guide to Community Preventive Services: What Works to Promote Health? [3]Promote evidence-based ways to deal with therapeutic and public health work on, making handy methods for sorting through the huge assortment of research is particularly essential since a significant part of the publichealth workforce is not generally all around prepared in how to fundamentally investigate the research evidence.

Establish the perimeter of biostatistics: Variation is flavor of life. This charming component in human beings is additionally a wellspring of huge vulnerabilities in clinical decisions. Other than genetic, biological, environmental, observer, instrumental and inspecting changeability, components, for example, deficient information on the patient, insufficiency of medicinal learning, poor consistence with the regimen, and defective medical tools add to the range of vulnerability. In some cases these vulnerabilities are profound to the point that medicine digresses from science to an art. Plainly, tools are required that assistance to keep an idea about these instabilities. They should be appropriately measured and techniques ought to be received to limit their effect on clinical decisions.

Role of Biostatistics in a Biomedical Research Center: Biostatistics applies theories and strategies of mathematics, probability theory, statistics, and computer science to logical issues in biomedicine. Its logical accomplices are intrigued to deliver, break down, and decipher quantitative information. They originate from biology, chemistry, pharmacology, epidemiology, genetics, genomics and medicine in general. Therefore, biostatistics interfaces factual science with biomedical science through statistical modeling and information examination for understanding test comes about. Biostatistics has been requested at less degree and infrequently by biomedical disciplines of a more distinct character, e.g. life systems, surgery or strong care of patients [4-6]. Most applicable for understanding the part of biostatistics has been the acknowledgment of biological variability. That was at that point communicated when Karl Pearson defined biometry as 'science which applies the current hypothesis of statistics to the investigation of variety and relationship in living structures'. Clarification of changeability using statistical modeling has dependably been a noteworthy assignment and it is one of best descriptors of a bio-statistician's work and his/her cooperation with biosciences with the point of view that 'the statistician going about as a researcher must have topic information. The part of biostatistics has been remarked in the same vain. Be that as it may, the part of a bio-statistical core unit in a clinical research focus has been tended to once in a while albeit self-origination and understanding one's position in science is exceedingly significant for a successful work in that environment [7-9]. Most regular and common obligations of a biostatistician in a biomedical environment are

- Design of experiments and clinical trials, calculation of sample size and statistical power

- Hypothesis testing and consulting therein in many variants

- Frequents (parametric or nonparametric) or Bayesian statistical inference

- Low- and high-dimensional regression analysis.

Biostatistics to Improve the Quality of Medical: The major applications of biostatistics started amidst the seventeenth century in the examination of key measurements. After the early improvements in imperative measurements, the field of hereditary qualities was the following territory that profited most from the new statistical ideas rising in progress of Charles Darwin. Presently, the fields of application and ranges of concern of biostatistics incorporate, among others, bioassay, demography, epidemiology, clinical trials, studies of human populations, group determination, bio-mathematical modeling, and so forth. Discoveries of good research 
should be introduced well, and a decent introduction is as much a piece of the examination as the careful gathering and investigation of the data. Critical commentators of the biomedical literature have reliably found that the greater part of the distributed articles (counting logical articles, distributed even in the best diaries) that utilized statistical methods contained unsuitable blunders. The term "statistics" here in this unique circumstance, has a more extensive importance and incorporates the procedure of research, study design, or epidemiological strategy and so forth. A recent study on the distributed writing of biomedical diaries has demonstrated that these blunders basically concern the specimen estimate, factual power, understanding amongst point and conclusion, dissemination of information, and additionally portrayal of area and changeability ofdata [10]. A short look through any recently medical statistical methods is assuming an inexorably noticeable part in present day medical research. At any rate, most research papers quote no less than one 'p-esteem' to convey. In the meantime, a developing number of papers are currently displaying the consequences of generally advanced, measurable investigations of complex arrangements of medical data. There are a few decent quality looks into detailed in restorative from creating areas without using the full discoveries of the review. Result part wound up noticeably poor as a result of the absence of learning in fitting test for the investigation of information and the coding of data. Medical Statistics helps the analyst to land at a logical judgment about a speculation. It has been contended that basic leadership is a necessary piece of a physician's work. Oftentimes, basic leadership is probability based.

Four Types of Bio-statistics in Bio-medicine: Next we discuss three frequently recuperated confusions on the capacity of a biostatistics unit in and outside a biomedical research center.

(i) Biostatisticians should work in the essential science department and not as an autonomous unit. Biostatisticians can work ideal just in an independent unit. The expert communication with other biostatisticians and the bolster which single biostatisticians get inside a departmental structure from the partner biostatisticians is a vital segment of the work itself and keeps her/him from getting to be noticeably separated or singlefollowed. The freedom of logical work in view of the standards depicted in the past segment is an essential to give sound and able measurable support for research.

(ii) Biostatistics is essentially required for information examination and it is required on a case by case premise. The parts most every now and again mistaken for that of an analyst have all the earmarks of being those of being a software engineer or database head. While a biostatistician, obviously, needs ability and involvement in those ranges keeping in mind the end goal to have the capacity to grow effectively statistical methods, these capacities can barely be essential to a statistician. Data generated by studies must be overseen and computer systems must be produced to encourage information gathering through included labor for programming, database creation and administration and adequate secretarial support. This work is however not quite the same as statistical thinking and the elaboration of statistical analyses which depend on scientific principles.

(iii) Biostatistics can work naturally or at request through software programs. This myth is once in a while situated with specialists not understanding that a few techniques (e.g. Cox relapse, blended impact models) have set aside a long opportunity to be created to the present state and can't - and even ought not-be connected by "running a program overnight" without a watchful bio-statistical analysis of the suppositions and characteristics in the information and the outcomes. Biostatisticians need some preparation to counter such contentions and to clarify the genuine way of statistics to biomedical researchers.

(iv) Bio-statistics is part of Bioinformatics. With the presence of sub-atomic succession information and microarray information and with the inherent issues of screening and chronicling these new and huge informational indexes developed in biomedicine the impression that bioinformatics tools would be the most suitable strategies to examine these information. Data mining and bunching techniques were overestimated in their intensity and computer programs were recently connected without a careful statistical analysis of the research problems and the properties of impromptu created optimization algorithms. In the perspective of some driving biomedical researchers biostatistics was considered as a major aspect of bioinformatics, if statistics was acknowledged at all as vital for the examination of the molecular data all in all and the genomic data specifically [11]. In spite of the fact that there were early notices this demeanor was not strong for pursuing bio-statistical research and benefit for plan and investigation of high-dimensional genomic data, and has really backed off the era of substantial biomedical results and sometimes biomedical research has even been misinformed by this misconception.

\section{Conclusion:}

The aim of this audit was to show that an intelligent idea of counseling, joint effort and research work can be sought after with accomplishment by a biostatistics department. Bio-statistical consulting and skill is critical for forming biometrical designs. Researchin displaying suggests the definition and approval of new plans for novel sorts of experiments and studies. We understood the need of congruity to cover both the standard fields of biostatistics (e.g. exploratory plan, disappointment time analysis, and non-parametric statistics) and 
developing fields (e.g. grouping, machine taking in, different examinations, contending dangers, and strategies for approval). We likewise need to stress that building and keeping up a biostatistics unit requires the vision to improve science through statistics and it requires a significant investment to set up a functioning biostatistics unit, in sharp complexity to the brief timeframe it might take to demolish it by misguided judgments and blunder. In study, one may state that a biostatistics unit/department

$>$ is active at the interface between the biomedical disciplines and the mathematical and statistical sciences and should add value to the quality of the center;

$>$ has to implement and foster continuously bio-statistical methods in the biomedical disciplines through service and research;

$>$ supports the center in total without giving preferences to some departments or groups;

$>$ Provides state of the art statistics, develops appropriate methods fit for purpose, disseminates and supports wide usage and it acts at national and international level.

\section{References:}

[1]. Sutherland, I. (1998): Medical Research Council streptomycin trial. In P. Armitage \& T. Colton (Eds.), Encyclopedia of Bio-statistics. Chichester, United Kingdom: John Wiley \& Sons, Ltd. (pp. 25592266).

[2]. Arndt, S., Woolson, R.F. (1991): Establishing a bio-statistical core unit in a clinical research center. The Amer. Statistician 45, 22-27.

[3]. Edler (2005): Computational Statistics und Biometrie: Wer treibt wen? Computational Statistics and Biometry. Which discipline drives which? GMS Med. Inform. Biom. and Epidemiol. 1, $27-43$.

[4]. Gehan, E.A. (2000): Bio-statistics in the new millennium: the consulting statistician's perspective. Stat.Meth. Med. Res 9, 3-16.

[5]. Ellenberg, J.H. (1990): Bio-statistical collaboration in medical research. Biometrics 46, 1-32.

[6]. Gehan, E.A. (2001): The role of the biostatistician in cancer research. Biom. Pharmacother. 55, 502-509.

[7]. Vingron M. (2001): Bioinformatics needs to adopt statistical thinking. Bioinformatics 17, 389-90.

[8]. Zaugg CE. (2003): Common bio-statistical errors in clinical studies; Schweiz Rundsch Med Prax;92(6): 218-224.

[9]. Glantz SA. (2009): Biostatistics how to detect, correct and prevent errors in the medical literature. Circulation; 61: 17.

[10]. Mittal A, Sathian B, Kumar A, Chandrasekharan N, Dwedi S. (2010): The Clinical Implications of Thyroid Hormones and its Association with Lipid Profile: A Comparative Study from Western Nepal. Nepal Journal of Epidemiology; 1(1): 11-6.

[11]. Mittal A, Sathian B, Chandrasekharan N, Lekhi A, Farooqui M S, Pandey N. (2011): Diagnostic Accuracy of Serological Markers in Viral Hepatitis and Non Alcoholic Fatty Liver Disease. A Comparative Study in Tertiary Care Hospital of Western Nepal. Nepal Journal of Epidemiology;1(2): 60-3.

Mobin Ahmad. "Impact of Bio-statistics in Medical Sciences." IOSR Journal of Dental and Medical Sciences (IOSR-JDMS) 16.7 (2017): 63-66. 